\title{
PART 4: DO WE NEED AQ? YES
}

Throughout this book, AQ is framed primarily as a type of communication and secondarily as a type of intelligence. The focus of this part of the book is to compare AQ directly to communication and intelligence theories. In evaluating a new theory against other theories, a simple criterion can be used; does the new theory add value to the existing theories? We choose a theory because it adds value compared to competing theories. According to one tally, there are 213 theories of communication. Cutting through this laundry list of theories, one way to examine the contribution of AQ to the communication perspective is to directly compare AQ to the sender-receiver model of communication. Introductory communication textbooks distill existing theory for a beginner's consumption, making decisions about what is most important that should be included, and what is omitted. In these textbooks, the senderreceiver model is foregrounded as the standard model of communication. The sender-receiver model is among the simplest communication theories and it underpins most other theories developed by academics and reflects the lay perspective upon the process of communication used daily in business, at home, and everywhere else in society. AQ is a theory of answers, and this perspective adds to our understanding of the sender-receiver model which focuses upon information exchange. When you started this book, you knew questions and answers were central to information exchange. AQ codifies this intuition as a theory of questions and answers. As this book comes full circle, this chapter brings into focus how AQ, as a theory of answers (and questions), can be added to our existing understanding of questions, to develop a fresh understanding of conversations. From the broadest vantage point, 
the value of $\mathrm{AQ}$ is to recast our understanding of the senderreceiver model, with emphasis upon communication as information exchange, and recast it as the conversational model of communication, with emphasis upon communication as a question and answer exchange.

The study of intelligence started with cognitive intelligence, and from there a proliferation of multiple intelligences have been advanced - emotional, practical, among others. I believe AQ fits with existing theories of intelligence. If you are smart (you have IQ). If you can perceive, understand, and regulate your emotions you have Emotional Intelligence (EQ). To have an impact, IQ and EQ need to be translated into answers that others can benefit from. 